\title{
Mehr als Macao: China in den portugiesischen Kulturzeitschriften Brasil - Portugal und O Occidente (1899-1901)
}

\author{
RICARDA MUSSER
}

„O que se está passando no Celeste Imperio não se pode ser indifferente a Portugal. Foram portuguezes os primeiros povos christãos que mantiveram relações com o extremo oriente e lá deixaram para sempre assignalada sua passagem” (,Was im Himmlischen Kaiserreich passiert, kann Portugal nicht gleichgültig sein. Die Portugiesen waren die ersten Christen, die Beziehungen mit dem fernen Osten aufnahmen und dort für immer die Zeichen ihrer Anwesenheit hinterlassen haben“) (O Occidente, 10.07.1900, S. 149). So war es in der Kolumne Chronik des Westens in der Kulturzeitschrift O Occidente vom 10. Juli $1900 \mathrm{zu}$ lesen. Um darzustellen, inwieweit sich das portugiesische Interesse an China in den Kulturzeitschriften Brasil - Portugal und O Occidente widerspiegelt, soll zunächst auf die portugiesische Präsenz in China sowie auf die kolonialen Interessen Portugals um 1900 eingegangen werden. Danach werden die beiden ausgewählten Kulturzeitschriften kurz vorgestellt und schließlich beschrieben, wie China in diesen dargestellt wurde.

Die Kontakte zwischen Portugal und dem Reich der Mitte gehen bis ins 16. Jahrhundert zurück. ${ }^{1}$ Bereits 1516 wurde eine Gesandtschaft zusammengestellt, die Kanton und Peking besuchte, vom Kaiser aber nicht empfangen wurde.

Die kantonesischen Behörden gestatteten den Portugiesen jedoch, sich in dem Fischerdorf A-Ma-Kao niederzulassen, wahrscheinlich als Belohnung für die Hilfe bei der Jagd nach Piraten.

Schon 1555 gab es dort eine kleine portugiesische Handelsniederlassung und eine Siedlung, die sich rasch entwickelte. Macao diente als Zwischenstation des Handels zwischen Japan und Europa. Fast zeitgleich gründeten portugiesische Händler Niederlassungen in anderen chinesischen Städten.

Macao wurde also nicht mit militärischen Mitteln erworben, sondern von China an Portugal verpachtet, das Tribut zahlte und gewisse Einmischungen der chinesischen Behörden akzeptierte. Der Gouverneur von Macao unterstand zunächst dem Vizekönig von Indien. Nach dem Vorbild von Lissabon wurde in Macao ein Stadtrat eingerichtet, der über lokale Fragen beriet. Da

1 Zur Geschichte des portugiesischen Kolonialreichs vgl. Marques. 
Macao dem Mutterland beträchtliche Gewinne einbrachte, verhielt sich die portugiesische Regierung tolerant gegenüber dem recht autonomen Agieren des Stadtrats und rührte auch nicht an dem politisch ungewöhnlichen Status des Gebiets. Der Handel zwischen Macao und Timor lag ebenfalls in den Händen des Rats und war bis ins 18. Jahrhundert hinein Haupteinnahmequelle für die Kolonie. Zu den wichtigsten Handelsgütern zählten Porzellan, Mobiliar, Sandelholz, Gold und Wachs. Weiterhin erzielte Macao hohe Gewinne mit der Verschiffung von Sklaven nach Amerika und Australien.

In den 60er Jahren des 16. Jahrhunderts entstand die Diözese von Macao, die, zumindest in der Theorie, fast ganz China umfaßte. Verschiedene religiöse Orden gründeten hier Niederlassungen, so die Franziskaner und die Jesuiten. Die erste Druckerei in der Kolonie wurde 1588 von Jesuiten eingerichtet. Es erschienen nicht nur Werke in portugiesischer Sprache, sondern auch in chinesisch.

Das ganze 19. Jahrhundert über konnte Portugal von der Schwäche Chinas profitieren und die uneingeschränkte Herrschaft über das kleine Gebiet ausüben. 1845 erklärte die portugiesische Regierung Macao zum Freihafen, was sich positiv auf das Handelsvolumen auswirkte. Ende des 19. Jahrhunderts wurde Macao auch von britischen und französischen Schiffahrtslinien angelaufen, die Europa mit Indien und China verbanden.

Der Gouverneur von Macao war zu dieser Zeit gleichzeitig auch portugiesischer Gesandter in China. Wenn es die Situation erforderte und spezielle diplomatische Missionen zu erfüllen waren, wurden von Lissabon mitunter Sondergesandte an den kaiserlichen Hof geschickt.

Portugal hatte in den letzten Jahrzehnten des 19. Jahrhunderts nicht die Möglichkeit, seinen Einflußbereich in China weiter zu vergrößern und sich beispielsweise an Minen- oder Eisenbahnunternehmen im Land zu beteiligen. Aus diesem Grund gehörte Portugal auch nicht zu den alliierten Mächten, die gemeinsam gegen die Boxer bzw. die chinesischen Truppen in den Kampf zogen.

Das portugiesische Kolonialreich hatte sich durch die Unabhängigkeit Brasiliens 1822 bereits dramatisch verkleinert. Der Fokus der portugiesischen Kolonialpolitik war Ende des 19. Jahrhunderts in dem Bestreben auf Afrika gerichtet, der Krone die Gebiete auf diesem Kontinent zu erhalten und möglichst zu erweitern. Der Krieg im Süden Afrikas beherrschte auch die Berichterstattung zu außenpolitischen Themen in der portugiesischen Presse um die Jahrhundertwende.

Um 1900 existierten in Portugal auf dem Literaturmarkt mehrere Kulturzeitschriften, deren gemeinsame Kennzeichen eine breite kulturelle Perspektive, die beispielsweise Literatur, Theater, Musik und Architektur umfaßte, und 
eine internationale Ausrichtung ${ }^{2}$ war. Trotz der peripheren Lage im äußersten Südwesten Europas zeigen die Kulturzeitschriften, daß Portugal im regen Informationsaustausch mit anderen Ländern Europas stand. Dies betrifft sowohl Fragen der Kultur als auch der aktuellen Politik. Brasil - Portugal erklärte diese Ausrichtung in der Ausgabe vom 14. Januar 1901 folgendermaßen:

É indispensavel a todo o momento completar o ponto de vista nacional pelo internacional. A explicação da politica local em cada nação moderna tem muitas vezes que procurar-se fóra das suas fronteiras. E sob este aspecto já não ha nações isoladas.

(Es ist unvermeidlich, der nationalen Sicht die internationale hinzuzufügen. Die Erklärung der lokalen Politik in einer jeden modernen Nation muß man sehr oft außerhalb ihrer Grenzen suchen. Unter diesem Aspekt gibt es keine isolierten Nationen mehr.). (Brasil - Portugal, 14.01.1901, o. Pag.)

Gleichzeitig wurden aber auch neuere und ältere literarische Erzeugnisse aus Portugal sowie Theateraufführungen im Land ausführlich besprochen. Obwohl einige Kulturzeitschriften bereits lange vor 1900 erschienen, kann man um die Jahrhundertwende eine Gründungswelle neuer Zeitschriften konstatieren. ${ }^{3}$ Als Verlagsorte der Kulturzeitschriften waren vor allem die Hauptstadt Lissabon und die zweitgrößte Stadt des Landes, Porto, von Bedeutung. Man kann von einer nationalen Verbreitung der einzelnen Kulturzeitschriften ausgehen, wenn sie in beiden Städten sowie in der Universitätsstadt Coimbra gelesen wurden. In diesen drei Städten war die Anzahl derjenigen mit einem mittleren oder höheren Bildungsabschluß am höchsten, aufgrund der zahlreichen in der Verwaltung, im Handel und an der Universität benötigten qualifizierten Personen. Die beiden ausgewählten Kulturzeitschriften sind heute in allen drei Städten in Bibliotheken nachweisbar. ${ }^{4}$

O Occidente mit dem Untertitel Illustrierte Zeitung von Portugal und vom Ausland (Revista ilustrada de Portugal e do estrangeiro) wurde in Lissabon zwischen 1878 und 1915 publiziert und erlebte insgesamt 1.314 Ausgaben in 34 Bänden (Pires, S. 223). Alle 10 Tage erschien ein neues Heft. Brasil - Portugal wurde als vierzehntägige illustrierte Zeitschrift ebenfalls in Lissabon herausgegeben. Sie existierte von 1899 bis 1914 (Pires, S. 89). Unter den in den beiden Kulturzeitschriften beschriebenen Ereignissen und Themen des Auslands zwischen 1899 und 1901 lag China vom Umfang her auf Platz drei hinter der Darstel-

2 Diese Ausrichtung hatten die portugiesischen beispielsweise mit den ebenso an der Peripherie gelegenen skandinavischen Kulturzeitschriften gemeinsam. Vgl. hierzu Paul, S. 77-78.

3 Einen sehr guten Überblick über die literarischen Zeitschriften unter Einschluss der Kulturzeitschriften in Portugal im 20. Jahrhundert gibt Pires; zu den Neugründungen um die Jahrhundertwende v.a. S. 37.

4 Nach Auskunft der Zeitschriftendatenbank ist O Occidente in Deutschland nur einmal nachgewiesen, nämlich in der Bibliothek des Ibero-Amerikanischen Instituts PK, Brasil - Portugal ist in keiner deutschen Bibliothek vorhanden. 
lung des Krieges im Süden Afrikas und der Weltausstellung in Paris. Insgesamt berichteten sowohl O Occidente als auch Brasil - Portugal auf jeweils 23 Seiten über China. Die Beschreibungen wurden durch Photographien und Zeichnungen ergänzt. Als Quellen der Informationen können die Times, der Standard und die Daily Mail, Telegramme aus London, Paris und Washington sowie in Macao lebende Portugiesen ausgemacht werden. In aller Regel wurden jedoch keine Quellen benannt. In der Darstellung bemühte man sich, die Fakten zu den Konflikten in China wahrheitsgemäß wiederzugeben; wenn aufgrund sich widersprechender Informationen Unsicherheiten bestanden, wurde dies thematisiert, ebenso, wenn sich Meldungen im Nachhinein als falsch erwiesen. So stand in O Occidente vom 30. Juli $1900 \mathrm{zu}$ lesen:

Verdade é que as notícias que chegam á Europa são o mais contradictorias que é possível. Depois das descripções dos morticinios commettidos pelos boxers em Pekim, notícias chegaram que dão como salvos muitos europeus e até alguns dos ministros, cujos necrologios foram publicados.

(Es ist so, daß die Nachrichten, die nach Europa kommen, so widersprüchlich wie nur möglich sind. Nach den Beschreibungen der Blutbäder, die die Boxer in Peking angerichtet haben, kamen Nachrichten, die davon sprechen, daß viele Europäer gerettet wurden, sogar einige Gesandte, deren Nekrologe bereits geschrieben worden waren.) (O Occidente, 30.07.1900, S. 165)

Es gibt nur eine Meldung, die man eher der Sensationspresse zuordnen könnte, nämlich die Beschreibung des Todes des deutschen Gesandten Klemens von Ketteler, der in seiner Sänfte von einem Angehörigen der chinesischen Armee erschossen wurde (vgl. Nowak, S. 116-117). In Brasil - Portugal vom 16. Juli 1900 wurde den Lesern jedoch folgender Tathergang geschildert:

Ei-lo montado no seu cavallo. Passeava pelas ruas de Pekim quando a furiosa multidão o agarrou, assassinando-o cruelmente, e martyrisando-o horrorosamente. Primeiro, arrancaram-lhe a lingua, depois fizeram-lhe saltar os olhos, para, acto continuo, o enterrarem até ao pescoço, quando ainda dava signaes de vida.

(Er befand sich auf seinem Pferd. Er war in den Straßen von Peking unterwegs, als die wütende Menge ihn ergriff und brutal ermordete und ihn so in schrecklicher Weise zum Märtyrer machte. Zuerst rissen sie ihm die Zunge heraus, danach die Augen, dann vergruben sie ihn bis zum Hals, und er zeigte immer noch Lebenszeichen.) (Brasil - Portugal, 16.07.1900, o. Pag.)

Die Themen, die in den beiden Kulturzeitschriften zu China behandelt wurden, lassen sich in vier Bereiche einteilen: erstens Portugal direkt betreffende Fragen im Zusammenhang mit China, zweitens die chinesische Kultur und die Sitten, drittens die Darstellungen der chinesischen Seite im Konflikt, nämlich Boxer, chinesische Armee und das Kaiserhaus, und viertens das Agieren der Alliierten während des Boxeraufstandes und während der Verhandlungen um das Boxerprotokoll. Alle vier Themenbereiche wurden in beiden Kulturzeitschriften berücksichtigt. Brasil - Portugal legte die Schwerpunkte auf Informati- 
onen zur chinesischen Kultur und zu den Friedensverhandlungen, während $O$ Occidente sich vor allem auf die Kriegshandlungen in China konzentrierte.

Der erste Bereich, Portugal direkt betreffende Fragen, nahm, da Portugal um 1900 den Fokus seiner kolonialen Interessen nicht auf China gerichtet hatte und nicht unter den Alliierten war, die in Peking in die Kämpfe involviert waren, nur relativ kleinen Raum ein. O Occidente informierte am 10. Juli 1900:

Os nossos direitos em Macau, que nos foi cedido pelos chinezes ha seculos, terão agora que ser mantidos, talvez pela força das armas. No dia 6 partiu para essa nossa possessão um punhado de valentes portuguezes que, se fôr preciso, hão de accrescentar mais uma folha de loiro á corôa opulenta [...]. El-rei e o sr. Infante D. Affonso foram despedir-se das tropas a bordo do Casengo. No mesmo vapor seguiu o novo governador de Macau, sr. Conselheiro Horta e Costa.

(Unsere Rechte in Macao, die uns von den Chinesen vor Jahrhunderten zugestanden wurden, werden jetzt verteidigt werden müssen, vielleicht mit Waffengewalt. Am 6. fuhr nach dieser unserer Besitzung eine Handvoll tapferer Portugiesen, die, wenn es nötig ist, unserer prächtigen Krone [...] ein weiteres Ruhmesblatt hinzufügen werden. Der König und Prinz Affonso haben sich von den Truppen an Bord der Casengo verabschiedet. Auf dem gleichen Dampfschiff befindet sich der neue Gouverneur von Macao, der Herr Rat Horta e Costa.) (O Occidente, 10.07.1900, S. 149-150)

Weitere Informationen und ein Bild des Gouverneurs erschienen in Brasil Portugal. Die Entsendung der Truppen war eine reine Vorsichtsmaßnahme, auf Macao griffen die Kämpfe nicht über. Am 14. Oktober 1901 erfuhr man aus Brasil - Portugal, daß die portugiesische Regierung José de Azevedo Castello Branco für eine diplomatische Mission nach China nominiert hatte.

O ministro de Portugal em Pekim é habitualmente o governador de Macau, mas, como já tem succedido varias vezes, a China furta-se sempre a tratar com esse funcionario portuguez questões diplomaticas, e agora que breve reunirá em Pekim uma conferencia internacional, com a assistencia de representantes de todas as nações, o governo portuguez entendeu que devia enviar ali um ministro extraordinario, tão importantes são os interesses de Portugal na Asia, sobretudo pelas proximidades em que as nossas colonias de Macau e Timor estão das fronteiras chinezas.

(Der portugiesische Gesandte in Peking ist üblicherweise der Gouverneur von Macao, aber wie es schon mehrfach passiert ist, hat sich China geweigert, mit dem portugiesischen Geschäftsträger diplomatische Fragen zu erörtern, und jetzt, wo sich in Peking bald eine internationale Konferenz versammeln wird, an der die Repräsentanten aller Nationen teilnehmen werden, hat die portugiesische Regierung entschieden, einen außerordentlichen Gesandten zu schicken, da die Interessen Portugals in Asien so bedeutend sind, besonders wegen der Nähe unserer Kolonien Macao und Timor zu den chinesischen Grenzen.) (Brasil - Portugal, 14.10.1901, S. 279) 
Das portugiesische Interesse richtete sich also auf die Sicherung der Besitzstände im asiatischen Raum.

Zum Themenbereich der chinesischen Kultur und Sitten begann Brasil Portugal am 16. Juli 1900 mit der Veröffentlichung einer Serie über diese Thematik. Im Zentrum stand dabei eine Reihe von Zeichnungen des USAmerikaners Shinery, der 1823-1838 in Macao und Hongkong gelebt hatte (vgl. Brasil - Portugal, 16.07.1900, o. Pag.). Die Skizzen zeigen dementsprechend Szenen aus Südchina. Die Notwendigkeit, die chinesische Kultur in den Mittelpunkt der Zeitschrift zu rücken und dem Lesepublikum näher zu bringen, wurde in der gleichen Ausgabe folgendermaßen begründet:

De ha muito que nos habituámos a vêr na China, atravez de um véo entretecido de lenda e de mysterio, o paiz nas suas grandezas de cunho exótico, de civilisação muito outra, mal podiamos comprehender se as coloridas descripções dos viajantes eram producto de escandecida imaginação ou realidade tangivel, tal é a variedade de quadros succedendo-se ininterruptamente no kaleidoscopo multicolor do fantastico e do imprevisto.

(Seit langer Zeit haben wir uns daran gewöhnt, China durch einen Vorhang aus Legenden und Mythen zu sehen, als das Land der Wunder. Gepriesen für seine Macht, unerklärlich in seiner Großartigkeit von exotischem Gepräge, in seiner so anderen Zivilisation, schlecht können wir ermessen, ob die bunten Beschreibungen der Reisenden ein Produkt überbordender Vorstellungskraft waren, oder sich auf die Realität bezogen, so weit gehen die Bilder jedenfalls auseinander, die sich ununterbrochen im vielfarbigen Kaleidoskop des Phantastischen und Unvorhergesehenen zeigen.) (Brasil - Portugal, 16.07.1900, S. 198)

Brasil - Portugal beschrieb für seine Leser im zweiten Halbjahr 1900 die chinesische Architektur, wie die Mauern und die Struktur von Peking, die chinesische Mauer, einen buddhistischen Tempel sowie ein Grabmal in Peking und hob dabei die außerordentlichen Fähigkeiten und Kenntnisse der Baumeister hervor. Weiterhin wurden in einem längeren, namentlich gekennzeichneten Artikel die religiöse Toleranz sowie die Leistungen im Bereich der Wissenschaft, Philosophie und Literatur gewürdigt:

A tolerancia religiosa, ao contrario do succedido em paizes que se dizem cultos, nunca teve límites n'um estado, que se dispensou sempre de religião official, nunca fechado ao budhismo, islamismo, judaismo e até ao proprio christianismo diffundido pelo portuguezes da colonia de Macau. [...] A febre do invento e dos seus aperfeiçoamentos [...] tem levado aos maiores prodigios a industria moderna, lá descobre-se a imprensa uns duzentos annos mais cedo que na Europa. [...] Elles conseguem ter uma litteratura colossal, em que avultam Confucio e muitos outros, não esquecendo o lendario $\mathrm{Fo} \mathrm{Hi}$, o mais antigo dos seus legisladores, todos elles manejando o mais espantoso e complicado de todos os alphabetos conhecidos.

(Die religiöse Toleranz, im Gegensatz zu dem, was in den Ländern passierte, die sich kultiviert nennen, war immer grenzenlos in einem Land, das niemals eine offizielle Religion hatte und sich nie dem Buddhismus, dem Islam, dem Judentum und sogar dem Christentum verschloß, das die Portugiesen in ihrer Kolonie Macao verbreiteten. [...] Das Fieber der Erfindung und deren Verbesserung [...] hat 
die größten Wunder der modernen Industrie hervorgebracht, man hat hier den Buchdruck mehr als zweihundert Jahre früher als in Europa entdeckt. [...] Sie haben eine kolossale Literatur, in der Konfuzius und andere hervorragen, um nicht den legendären Fo Hi zu vergessen, den ältesten der Gesetzgeber, alle benutzten das erstaunlichste und komplizierteste aller bekannten Alphabete. (Brasil - Portugal, 16.07.1900, S. 198-199)

Die positive Wertung der chinesischen Kultur war in allen Texten zu diesem Bereich in den beiden Kulturzeitschriften zu erkennen. Vergleiche zwischen der Zivilisiertheit Chinas und dem sich als zivilisiert gebenden Abendland kommen an verschiedenen Stellen vor, so wie im genannten Zitat hinsichtlich der religiösen Toleranz, aber auch im Hinblick auf die Zerstörung von Zeugnissen der chinesischen Baukunst durch die Europäer und auf die Art ihrer Kriegsführung. Sie fallen in der Regel zuungunsten Europas aus, das sich in den Augen der Autoren der portugiesischen Kulturzeitschriften auf keinen Fall als Kulturbringer und Förderer des Fortschritts positioniert. Portugal selbst allerdings wurde von der Kritik im wesentlichen ausgenommen. Dies erkennt man an Formulierungen wie ,de meado do seculo XIX, para cá [...] a perseguição a missionarios, que não são nossos, começou.“ (,,von der Mitte des 19. Jahrhunderts an bis jetzt [...] begann die Verfolgung der Missionare, die nicht die unseren waren.") (Brasil - Portugal, 16.07.1900, S. 198)

Wenn aber Macao und das Leben in der portugiesischen Kolonie beschrieben wurde, änderten sich jedoch die positiven Wertungen dahingehend, daß die dort lebenden Chinesen als betrügerisch und abergläubig, sowie in Kenntnissen und Bräuchen weit entfernt von der ansonsten bewunderten chinesischen Hochkultur dargestellt wurden:

Scena domestica. Lá está o meu cosinheiro [...] lá está elle rezando aos seus deuses protectores. Que lhe preste. Acabou de me roubar nas contas, como bom chinez que é, serenamente agressivo em tudo ao europeu; e passou a entregar-se a esta outra occupação não menos meritoria. Sendo seus os aposentos inferiores, é alli rei, o pelo menos mandarim; faz o que quer. [...] Alli ardem lumes mysticos; e frequentemente pela noite, como agora, se queimam pivetes, cirios rubros, rezinas e papeis, de tudo emanando um fumo atroz, que invade em torvelino a casa todas, que chega sem respeito ao sitio onde me encontro, e me suffoca. Paciencia. Paciencia, é o unico codigo de conducta para o aventureiro, que escolheu para exilio um canto exotico, longe, muito longe do torrão onde nasceu [...] Os deuses [...] e a cuja protecção, posto que indirectamente, me confio, são muitos um enxame. É todo o Olympo buddhista e o inteiro mytho primitivo, amalgamados em crendices.

(Eine häusliche Szene. Da ist mein Koch [...] wie er zu seinen Schutzgöttern betet. Auf daß es ihm nütze. Er hat mich gerade mit den Abrechnungen betrogen, als guter Chinese, der er ist, gemäßigt aggressiv gegenüber allem Europäischen, und er hat sich nun dieser anderen, nicht weniger verdienstvollen Tätigkeit zugewandt. Ihm gehören die hinteren Zimmer, dort ist er König, oder zumindest Mandarin; er macht dort, was immer er will. [...] Dort brennen mystische Lichter; und oft in der Nacht, so wie jetzt, brennen Räucherkerzen, glühendrote Altarker- 
zen, Gewebe und Papier, und alles verströmt einen gräßlichen Rauch, der sich in Wirbeln im ganzen Haus verbreitet, der auch respektloser Weise dort hinkommt, wo ich mich befinde, und mich erstickt. Geduld. Geduld ist der einzige Verhaltenskodex für den Abenteurer, der sich zum Exil eine exotische Ecke, weit, sehr weit entfernt von dem Land seiner Geburt ausgesucht hat [...] Götter [...] in deren erhabenen Schutz man indirekt kommt, gibt es in großer Menge, wie ich bestätigen kann. Es ist der ganze buddhistische Olymp und der gesamte primitive Mythos, vermischt im Aberglauben.) (Brasil - Portugal, 30.07.1900, S. 274)

Im nächsten Themenbereich, den Darstellungen der chinesischen Seite des Konflikts, informierte O Occidente am 20. Juni 1900, daß die aufständischen Boxer circa 100.000 an der Zahl seien und daß ihr Anführer Chan sich selbst zum Kaiser proklamieren lassen möchte, weiterhin, daß die Rebellen versuchten, die Christen auszurotten und die alten Gewohnheiten des Kaiserreichs wiederherzustellen. Dazu wurde gemeldet, daß die französischen und englischen Missionen in Yunnam angezündet wurden, ebenso wie die Sommerresidenzen der Gesandtschaften im Westen von Peking und die katholische Kathedrale im Osten der Stadt. Im Hinblick auf die Schlagkraft der chinesischen Armee wurde am 10. September 1900 in O Occidente mitgeteilt:

Quem, pela discripção, que dos soldados chinezes se fazia ha meio seculo, quizesse formar hoje idea do exercito do Celeste Imperio, muito por certo se enganaria. Já vae longe o tempo em que os chinezes cuidavam pôr em debandada o inimigo, fazendo grandes berrarias e mostrando-lhe horrorosos monstros pintados. Sem falarmos da marinha chineza, que possue navios modernos de primeira ordem, basta-nos dar conta succinta dos seus armamentos em terra para que fique demonstrado se teem ou não razão os que tanto se esforçam por mostrar a grandeza do perigo amarello. Desde fevereiro de 1898 que os chinezes possuem oitenta e quatro canhões de tiro rapido, construidos nas officinas de Krupp, bem como cento e oitenta canhões de tiro rapido sahidos dos officinas do Creusot. Além d'este armamento [...] a China possue muitos outros excellentes canhões de differentes systemas todos manejados por pessoal muito instruido, como ficou provado nos ultimos combates. Quasi a terça parte do mundo é chineza. Os soldados são todos valentes e teem o maior despreso pela morte. Nenhum paiz pode como a China levantar um exercito poderoso. Poderá portanto combater contra a Europa não só pela industria, o que ha muito andava fazendo, mas até pelas armas. O perigo amarello é portanto um serio e verdadeiro perigo.

(Wer, durch die Beschreibung, die von den chinesischen Soldaten vor einem halben Jahrhundert gemacht wurde, sich heute eine Vorstellung von der Armee des Himmlischen Kaiserreiches machen wollte, würde sich mit Sicherheit sehr irren. Die Zeit ist lange vorbei, in der die Chinesen ihre Feinde mit großem Gebrüll in die Flucht schlagen wollten, oder ihnen Bilder von schrecklichen Monstern zeigten. Ohne von der chinesischen Marine zu sprechen, die moderne Schiffe erster Ordnung besitzt, genügt es, kurz von den Waffen an Land zu berichten, um zu zeigen, ob diejenigen, die von der gelben Gefahr sprechen, Recht haben oder nicht. Seit Februar 1898 besitzen die Chinesen 84 Schnellfeuerkanonen aus der Firma Krupp sowie 180 Schnellfeuerkanonen der Firma Creusot. Außer dieser Bewaffnung [...] besitzt China viele andere exzellente Kanonen verschiedener Systeme, 
die alle von gut ausgebildetem Personal bedient werden, wie sich in den letzten Schlachten gezeigt hat.

Fast ein Drittel der Welt ist chinesisch. Die Soldaten sind alle mutig und zeigen die größte Todesverachtung. Kein Land könnte wie China eine solch machtvolle Armee aufstellen. Das Land könnte gegen Europa kämpfen, nicht nur in der Industrie, wie es das bereits tut, sondern auch mit den Waffen. Die gelbe Gefahr ist eine ernste und wirkliche Gefahr.). (O Occidente, 10.09.1900, S. 198-199)

Diese Aussagen erschienen interessanterweise erst, nachdem Peking bereits seit einem Monat von den Alliierten erobert worden war.

Den größten Umfang zur Berichterstattung über China nahm in den portugiesischen Kulturzeitschriften das Agieren der Alliierten während des Boxeraufstandes und der Verhandlungen um das Boxerprotokoll ein. Die Darstellungen darüber begannen nach dem 20. Juni 1900, also nach der Ermordung des deutschen Gesandten Klemens von Ketteler. Im Jahre 1900 dominierten in den beiden Kulturzeitschriften historische Rückgriffe auf den Zweiten Opiumkrieg $^{5}$ und auf die Vorstellung einzelner Persönlichkeiten, die in die Kämpfe auf Seiten der Alliierten involviert waren, wie des britischen Gesandten und des Admirals Kempff, der die nordamerikanischen Seestreitkräfte befehligte. Interessiert verfolgten die Kulturzeitschriften weiterhin die neuen Allianzen, die der Konflikt in China begünstigte, wie zum Beispiel: „Mas que voltas dá o mundo! Lá vão soldados allemães bater-se ao lado dos francezes!“ („,Wie sich die Welt verändert hat! Da werden deutsche Soldaten an der Seite der Franzosen kämpfen!“") (O Occidente,10.07.1900, S. 149) Mit großer Aufmerksamkeit beobachteten die Kulturzeitschriften die Friedensverhandlungen und die Vorbereitung eines Vertrages mit China. So konstatierte Brasil - Portugal am 14. Januar 1901:

O paiz classico [...] do odio ao estrangeiro, está convertida no movimentado theatro de todas as ambições europeias, e póde affirmar-se [...] que mais do que em Pekin ou no valle do Yang-tse-kiang, o destino historico d'esta desditosa nação está sendo jogado nas chancellarias das grandes potencias.

(Das klassische Land [...] des Hasses auf das Ausland ist zu einem bewegten Theater der europäischen Ambitionen geworden, und man könnte sagen, [...] daß sich im Hinblick auf Peking oder auf das Yang-tse-kiang-Tal das historische Schicksal dieser unglücklichen Nation in den Kanzleien der Großmächte entscheidet.) (Brasil-Portugal, 14.01.1901, o. Pag.)

Mehrfach wurde auf die Möglichkeit des Aufbrechens von Konflikten zwischen den Alliierten hingewiesen, wie zum Beispiel zwischen Rußland und Großbritannien in der Frage der Mandschurei und zwischen Japan einerseits und Deutschland und Großbritannien andererseits im Hinblick auf die staatliche Souveränität Chinas. Am 29. August 1901 meldete Brasil - Portugal:

5 Vgl. hierzu Mühlhahn. 
Depois de longas e trabalhosissimas negociações chegaram finalmente as potencias alliadas a um accordo com a China, que por agora vem pôr termo ao estado de meia guerra em que este paiz, depois do ataque ás legações, se conservava com a Europa. O que este accordo valerá no futuro ninguem o pode prevêr. [...] Os crimes que motivaram a intervenção ficaram na quasi totalidade impunes, pelo menos a respeito dos principaes responsaveis, que a estas horas se estão rindo da ingenuidade das nações do occidente. A côrte continúa a estar ausente de Pekin, quer dizer, fóra da acção e da influencia dos ministros extrangeiros. Os boxers, longe de terem sido anniquilados, reconstituem-se nas differentes provincias do imperio, preparando, segundo todas as probabilidades, para proximo futuro tremenda desforra ao cheque agora soffrido. [...] Se ao menos a Europa podesse inscrever ao seu activo qualquer aumento de prestigio ou de força moral proveniente da desastrada intervenção, que a furia guerreira de Guilherme II tornou inevitavel, ainda alguma cousa teria ganho a civilisação do occidente. [...] Com effeito, que ideia estarão fazenda actualmente os chinezes da superioridade de uma civilisação que, em pleno seculo XX, a titulo de vingar as offensas commettidas por um bando de criminosos irresponsaveis e anonymos, assassinou a sangue frio, obedecendo à palavra d'ordem que trazia de Hamburgo, mulheres, crianças, velhos, trucidando sem piedade a população inerme, talando e incendiando, como se quizesse adrede ressuscitar os crueis processos da guerra medieval? Triste exemplo de decadencia moral foram as nações christãs levar á China pagã.

(Nach langen und arbeitsreichen Verhandlungen sind die alliierten Mächte schließlich zu einer Übereinkunft mit China gekommen, die zum Ziel hat, den Krieg zu beenden, in dem sich das Land seit dem Überfall auf die Botschaften mit Europa befindet. Was dieser Vertrag in Zukunft wert sein wird, ist schwer vorauszusehen. [...] Die Verbrechen, die Ursache der Intervention waren, sind fast völlig ungestraft geblieben, wenigstens im Hinblick auf die Hauptverantwortlichen, die jetzt über die Naivität der Völker des Westens lachen. Der Hof hält sich weiterhin fern von Peking auf, das heißt, fern von den Aktionen und dem Einfluß der ausländischen Gesandten. Die Boxer, weit davon entfernt, vernichtet zu sein, konstituieren sich in den verschiedenen Provinzen des Kaiserreichs neu, und bereiten, nach aller Wahrscheinlichkeit, für die nächste Zukunft die Rache für das erlittene Mißgeschick vor. [...] Wenn Europa wenigstens auf der positiven Seite irgendeine Erhöhung seines Prestiges oder der moralischen Kraft aus der unseligen Intervention ziehen könnte, die die Kriegsfurie von Wilhelm II. entfesselt hat, hätte die westliche Zivilisation wenigstens etwas gewonnen. [...] Das heißt, welche Vorstellung werden sich wohl die Chinesen von der Überlegenheit einer Zivilisation machen, die am Anfang des 20. Jahrhundert im Namen der Rache an einer Bande verantwortungsloser und anonymer Krimineller, kaltblütig Frauen, Kinder und Alte ermordete, dem Befehl aus Hamburg ${ }^{6}$ gehorchend, die wehrlose Bevölkerung ohne Mitleid niedermetzelte, Zerstörungen anrichtete und brandschatzte, als wollte sie die Brutalitäten des mittelalterlichen Krieges wiederbeleben? Ein trauriges

6 Hierbei handelt es sich um eine Anspielung auf die „Hunnenrede“, die Wilhelm II. anläßlich der Verabschiedung der deutschen Truppen nach China gehalten hatte, diese aber in Bremerhaven und nicht in Hamburg. 
Beispiel des moralischen Niedergangs gaben die christlichen Nationen im heidnischen China.) (Brasil - Portugal, 29.08.1901, o. Pag.)

Am 28. September 1901 wurde schließlich, um bei den traurigen Beispielen zu bleiben, die Sühnemission Chinas in Deutschland beschrieben. Bereits in vorangegangenen Artikeln war deutlich geworden, daß die Politik Deutschlands gegenüber China dezidiert kritisch bewertet wurde:

A Allemanha ter commettido uma grande falta. É sabido que uma das condicções impostas por Guilherme II para firmar a paz com a China, foi de que viria a Berlin uma missão expiatoria [sic!]para perante o imperador se desculpar solemnemente do assassinato do barão de Ketteler. Até aqui não ha muito que objectar, attendendo sobretudo á maneira espectaculosa como o imperador allemão costuma cercar os actos ás vezes ainda os mais insignificantes da sua política. A missão veio, por tal signal presidida pelo principe Tahung, irmão do proprio filho do Ceo. Como se esta humilhação não fosse, porém, ainda bastante para satisfazer o seu orgulho, Guilherme II exigio que perante elle o embaixador chinez e o seu sequito prestassem a homenagem do kotau, isto é, batessem, depois de um certo numero de reverencias, tres vezes com a fronte no chão. [...] O principe Tchung recusou. [...] Por fim a Allemanha teve de ceder, contentando-se Guilherme II em preparar para a recepção da missão expiatoria uma mise-en-scène, que devia ter feito sorrir o astuto enviado oriental, que afinal foi quem ganhou a partida. [...] Emfim, pôde bem dizer-se que em todo este triste negocio chinez e desde o começo d'elle a Europa não fez mais do que proceder contra o seu proprio interesse, umas vezes com uma miopia e leviandade indisculpaveis, outras com uma crueldade incompativel com os principios que affirmava ir defender ao Oriente, e por ultimo para completar a sua obra de insania, com uma inhabilidade que em algumas horas lhe fez perder o resto da força moral, que ainda podia conservar aos olhos dos chinezes.

(Deutschland hat eine große Schuld auf sich geladen. Es war bekannt, daß eine der Bedingungen, die Wilhelm II. diktiert hatte, um den Frieden mit China zu unterzeichnen, diejenige war, daß eine Sühnemission [sic!] nach Berlin kommen sollte, um feierlich für die Ermordung des Barons von Ketteler um Entschuldigung zu bitten. Bis hierher kann man nicht viel einwenden, wenn man vor allem die theatralische Art berücksichtigt, in der der deutsche Kaiser normalerweise mit Vorgängen umgeht, die von wesentlich geringerer Bedeutung für seine Politik sind. Die Mission kommt und wird von Prinz Chung angeführt, einem Bruder des Sohnes des Himmels, als ob diese Demütigung nicht reichen würde, und um seinen Stolz zu befriedigen, forderte Wilhelm II., daß der Chinesische Botschafter und sein Gefolge vor ihm die Ehrung des Kotau ausführen, das heißt, nach einer gewissen Anzahl von Verbeugungen mit der Stirn drei Mal auf die Erde schlagen. [...] Prinz Chung hat dies abgelehnt. [...] Schließlich mußte Deutschland nachgeben, Wilhelm II. gab sich beim Empfang der Sühnemission mit einer Inszenierung zufrieden, die dem schlauen Orientalen gefallen haben muß, denn schließlich war er derjenige, der die Partie gewonnen hatte. [...] Schließlich könnte man sagen, daß [Wilhelm II.] in dieser ganzen traurigen chinesischen Angelegenheit und seit deren Beginn in Europa, nichts anderes getan hat, als gegen seine eigenen Interessen zu handeln, einige Male mit unentschuldbarer Kurzsichtigkeit und unentschuldba- 
rem Leichtsinn, andere Male mit einer unvergleichlichen Brutalität gegenüber den Prinzipien, die er im Orient zu verteidigen angetreten war, und schließlich, um seinen Wahnsinn komplett zu machen, mit einer Unfähigkeit, die ihn zuzeiten den Rest der moralischen Kraft verlieren ließ, die er sich noch in den Augen der Chinesen bewahrt hatte.) (Brasil - Portugal, 28.09.1901, o. Pag.)

Möglicherweise erklärt sich die sehr kritische Haltung gegenüber der deutschen Position auch daraus, daß Portugal im Hinblick auf die kolonialen Interessen in Afrika erheblich von Deutschland bedrängt wurde, nämlich im Norden von Moçambique und im Süden von Angola.

Zusammenfassend kann gesagt werden, daß in den portugiesischen Kulturzeitschriften herausgestellt wurde, daß Portugal, das seit Jahrhunderten als Kolonialmacht in Asien und auch in China präsent war, nicht in den Boxeraufstand involviert war, sondern den Konflikt als außen stehender Beobachter wahrnahm. Die eigene Rolle in China wurde dabei positiv und in Abgrenzung zur Handlungsweise der Alliierten dargestellt. Für die Chinesen und ihre Kultur wurde mehrfach Partei ergriffen. An den Alliierten, zuvorderst an Deutschland, wurde verschiedentlich Kritik geübt. Diese bezog sich vor allem auf die Art der Kriegsführung, bei der auf Schätze der Kunst und Kultur und auf die Zivilbevölkerung keinerlei Rücksicht genommen wurde. Damit spiegelte sich die Betrachtungsweise der portugiesischen Politik in den Kulturzeitschriften um die Jahrhundertwende wider.

\section{Anhang}

Brasil - Portugal

16.07.1900 o.Pag. - Anon.: Os acontecimentos da China. O barão de Ketteler 16.07.1900 198-199 - L.F. Marrecas Ferreira: O povo chinez 30.07.1900 274-275 - Wenceslau de Morais: Pau-Man-Chen 14.01.1901 o.Pag. - Consiglieri Pedroso: Política Internacional 29.08.1901 o.Pag. - Consiglieri Pedroso: Política Internacional 28.09.1901 o. Pag. - Consiglieri Pedroso: Política Internacional 14.10.1901 279 - Anon.: Missão á China

O Occidente. Revista Illustrada de Portugal e do Extrangeiro 10.07.1900 149-150 - João da Câmara: Chronica Occidental 30.07.1900 165-166 - João da Câmara: Chronica Occidental 10.09.1900 198-199 - Anon.: Os acontecimentos na China. O exercito chinez 


\section{Literaturverzeichnis}

Marques, António Henrique de Oliveira: Breve História de Portugal, Lisboa (Presença) 1995.

Mühlhahn, Klaus: China und der westliche Imperialismus, in: Leutner, Mechthild / Mühlhahn, Klaus (Hg.): Kolonialkrieg in China. Die Niederschlagung der Boxerbewegung 1900-1901, Berlin (Links) 2007, 15-26.

Nowak, Dominik: Der Tod des deutschen Gesandten Clemens von Ketteler, in: Leutner, Mechthild / Mühlhahn, Klaus (Hrsg.): Kolonialkrieg in China. Die Niederschlagung der Boxerbewegung 1900-1901, Berlin (Links) 2007, 111-117.

Paul, Fritz: Skandinavische Kulturzeitschriften um 1900, in: Europäische Kulturzeitschriften um 1900 als Medien transnationaler und transdisziplinärer Wahrnehmung. In $\mathrm{Zu}-$ sammenarbeit mit Susanne Friede hg. von Ulrich Mölk, Göttingen (Vandenhoeck \& Ruprecht) 2006 (Abhandlungen der Akademie der Wissenschaften zu Göttingen, Philologisch-Historische Klasse, Dritte Folge, Band 273), 77-91.

Pires, Daniel: Dicionário das Revistas Literárias Portuguesas do Século XX, 1986. 
